\title{
Interactive comment on "Case Studies of the Impact of Orbital Sampling on Stratospheric Trend Detection and Derivation of Tropical Vertical Velocities: Solar Occultation versus Limb Emission Sounding” by L. F. Millán et al.
}

\section{Anonymous Referee \#2}

\section{Received and published: 17 May 2016}

This paper analyzes the impact of orbital sampling on remotely sensed data and investigates implications of related sampling biases and drifts. Although this is a methodical and partly technical paper, I recommend publication in ACP rather than any of the more technical journals, because the readership addressed are scientists working in atmospheric sciences rather than scientists working in more technical fields.

The methodology chosen is adequate; results are interesting; the presentation is concise without any unnecessary length but still complete; the authors have taken care to put their work adequately into the context of existing work. I recommend publication in 
ACP and I have only a list of minor comments which may help to improve the paper.

p.2 124-32: While I usually insist that results must be summarized in the abstract (as ACPD correctly done in this paper), I am not sure if it is adequate to summarize the results already in the introduction. Usually it is announced in the introduction what will be investigated, without anticipating the results.

p3 118: It should probably read "long-term $\mathrm{O}_{3}$ trends" here?

Interactive

comment

p4 14: I miss some information about the HALOE antitude resolution here.

p4 19: Same for ACE-FTS.

p4 113: Same for MLS.

p4 121: It might not even be necessary to assume that the vertical resolutions are the same. If the true fields are smooth enough in the vertical domain, even different vertical resolutions cause only marginal differences. Possibly the assumption of equal vertical resolutions can be challenged, and since a much weaker assumption (that all vertical resolutions are good enough to resolve the fields) might be sufficient, I suggest to formulate the argument with this weaker assumption.

p5 112-22: Some discussion of the distributions of the biases might be useful. Where are the largest biases and why? Is this consistent with Toohey et al.?

p4 122: On page 3 it is not stated how fine the vertical sampling of the model is in the lower and middle stratosphere but I guess it is much better than the vertical resolution of ACE-FTS, which is often reported to be around $3 \mathrm{~km}$, if I remember correctly. Unfortunately to my knowledge neither for ACE-FTS nor for HALOE averaging kernels are available. But if you smooth the model fields using a triangular or Gaussian function of $3 \mathrm{~km}$ full width at half maximum, and if the model fields change only marginally by this, then you are on the safe side. At least Fig 2. suggests that there seem to exist no major vertical resolution related problems.

Printer-friendly version

Discussion paper 
p5 I25 Temperature: why capital "T"?

p6 14: RMS and standard deviation are often not adequately distinguished, and I am not sure if centered RMDd is a widely known concept. I would not mind to see these terms defined by equations here.

p6 I5 "everything" sounds a bit vague. "all data" or something similar would sound a bit more "intellectual" but would say essentially the same.

p6 I15 shows the raw model (remove one "the") .

p7 11: It would be interesting to know if the autocorrelation is assumed constant over the entire time series or if it has som time-dependence in itself.

p7 14: It would be interesting to see if the sampling artefacts somehow show up as autocorrelation. Can we learn anything about this by analysis of the $\phi$ values of the various instruments?

p7 110: Trends in state variables can have latitudinal structure (see, e.g. Eckert et al., ACP, 2014, for ozone). I do not challenge the adequacy of trend analysis in more global terms but a caveat about latitudinal structure might be useful.

p7 121-23: I am not happy with the term "noise" here, because this can easily be understood as "measurement noise". But here the residuum is meant, which contains real physical effects, like QBO or whatever. I suggest "The autocorrelation of the residual between the data points and the trend model" ... "The standard deviation of the residual, which corresponds ..."

17 125: I do not like the term "model" here, because there are so many models (trend models, CMAM, etc). I would prefer a more specific term here.

Printer-friendly version

p8 110-12: The dichotomy "solar occultation vs. microwave emission" does not exist. The HALOE and ACE-FTS data gaps due to clouds are due to the spectral region (IR), not to the measurement geometry (occultation). The IR instrument MIPAS, e.g., has 
also data gaps due to clouds although it is an emission instrument. The statement made here is certainly correct (except for the dichotomy-like wording) but it seems to me to have nothing to do with orbital sampling. I think that the discussion of this issue detracts from the main issue of this paper. If you want to discuss sampling artefacts due to clouds, the fact that IR sounders have a sampling bias towards the cloud-free atmosphere would be interesting and deserves an investigation, particularly in the context of water vapor, but this is clearly beyond the scope of this paper.

Sect 4 general: Unless I have got things completely wrong, the following question remains unanswered: MLS provides both denser sampling and more uniform sampling. Both characteristics contribute to the better MLS trends. Which of the sampling attributes (data density or uniform sampling) is the primary cause of the better MLS trends? Could analysis of the $\phi$ values help to find this out?

If I haven't missed anything, the data points have equal weight in the trend fit. Is this adequate? Since orbits converge with higher latitudes, each data point represents a smaller area at higher latitudes. Thus, high latitudes are over-represented if no areaweighting is applied. This, however, is a kind of sampling bias in itself.

p9 I13 ff: there appear a lot of abbreviations and acronyms (QBO, ENSO, QSI, MEI). Please make sure that they all are defined. Some of them are but I have not checked all of them.

p10 126: Here the better performance of instruments with MLS sampling pattern is attributed to the data density. This is intuitive but I am not sure if it has really been shown that this is not due to uniformity. The $\phi$ is not necessarily the same for the instruments under investigation.

p11 17: This reads a bit as if the increase in the circulation is a fact but to my knowledge it is an expectation based on model calculations. To my knowledge the increase of circulation has not yet been empirically confirmed, at least not beyond any doubt. I would prefer "such as a possible increase in the circulation". For my personal taste, 
the last three lines are a bit too general and I would prefer a concluding remark which is more focused on the sampling issue.

p11 110: Finally a fussy one: (Do not take it too seriously because as a non-native English speaker I may be wrong here) It sounds funny to me that a laboratory does the work. I thought it is the researchers who do the work. The researchers are listed in the authors' list, along with their affiliations. Thus it seems redundant to me to mention this again in the acknowledgements.

Fig Cap 5: "either K or \%" sounds a bit vague. Better: ...as a function of pressure for temperature (in Kelvin) and $\mathrm{O}_{3} \ldots$ (in \%). Again: why capital "T" for temperature"?

Interactive comment on Atmos. Chem. Phys. Discuss., doi:10.5194/acp-2016-356, 2016. 